\title{
Tratamento da cólica infantil: uma revisão baseada na evidência
}

Ana Clara Moreira, ${ }^{1}$ Joana Catarina Castro, ${ }^{1}$ Joana Filipa Barreira, ${ }^{1}$ Raquel Coimbra ${ }^{2}$

\section{RESUMO}

Objetivos: O objetivo é rever a eficácia das intervenções terapêuticas na cólica infantil, uma vez que se trata de um motivo frequente de consulta, o tratamento é difícil e constitui motivo de frustração para pais e profissionais de saúde.

Fontes de dados: Bases de dados National Guideline Clearinghouse, Canadian Medical Association Practice Guidelines Infobase, NICE, Cochrane, DARE, Bandolier e MEDLINE.

Métodos de revisão: Pesquisa de meta-análises (MA), revisões sistemáticas (RS), ensaios clínicos aleatorizados e controlados (ECAC), normas de orientação clínica (NOC) e outros estudos originais, em português, inglês e espanhol, publicados entre janeiro de 2006 e outubro de 2016, utilizando o termo MeSH infantile colic. Foi utilizada a escala de Jadad para avaliar a qualidade dos ensaios clínicos e a escala Strength of Recommendation Taxonomy (SORT) para atribuição do nível de evidência e força de recomendação.

Resultados: Dos 185 artigos obtidos, 17 preencheram os critérios de inclusão (quatro MA, sete RS, três ECAC e três NOC). Na sua maioria, o outcome avaliado foi o tempo de choro. As fórmulas hidrolisadas demonstraram eficácia; porém, não devem ser utilizadas indiscriminadamente. A evidência aponta-se mais consistente no uso de probióticos ( $L$ reuteri DSM 17938), havendo redução do tempo médio de choro diário, em regime de prevenção ou tratamento. O simeticone, frequentemente utilizado, não revelou benefícios. Nas terapêuticas complementares existe alguma evidência a favor da fitoterapia à base de funcho.

Conclusões: Existem algumas estratégias que parecem apresentar benefício no tratamento da cólica infantil; no entanto, a evidência é escassa e pouco consistente. São necessários mais estudos com amostras maiores e critérios de diagnóstico padronizados. Tendo em conta que é um processo autolimitado, na ausência de evidência robusta sobre a eficácia dos tratamentos, a atitude expectante pode ser uma estratégia a considerar.

Palavras-chave: Cólica infantil; Recém-nascido; Lactente; Cuidados de saúde primários.

\section{INTRODUÇÃO}

A cólica infantil afeta $10-40 \%^{1}$ das crianças nos primeiros meses de vida, tendo a sua maior expressão entre as seis e oito semanas e resolvendo espontaneamente por volta dos três-quatro meses. Constitui $10-20 \%^{2}$ dos motivos de consulta nas crianças nesta faixa etária, sendo um importante motivo de preocupação dos pais.

Apesar da sua benignidade, os resultados com o tratamento são dúbios e a busca por respostas condiciona o uso de fármacos, alterações alimentares, ansiedade e exaustão parental, levando a estados de frustração

1. USF Viver Mais, ACeS Maia/Valongo.

2. USF Luz, ACeS Lisboa Norte. nos pais e profissionais de saúde. ${ }^{3}$ Alguns estudos indicam que as consequências psicossociais podem ser várias, apontando mesmo uma associação entre cólica infantil e depressão materna ${ }^{4}$ ou abuso infantil (shaken baby syndrome). ${ }^{5}$

Os sintomas são heterogéneos, podendo ir desde flatulência excessiva, inquietação ou irritação sem causa aparente, sinais de dor (e.g., flexão dos joelhos ou hiperextensão do tronco) ou mesmo choro frequente. De forma a obter consenso, Wessel definiu como critérios de diagnóstico a presença de episódios de choro paroxístico, com duração de pelo menos três horas por dia, por mais de três dias da semana, durante pelo menos três semanas, em crianças previamente saudáveis e bem alimentadas. ${ }^{6}$ 


\begin{tabular}{|c|c|c|c|c|}
\hline \multicolumn{5}{|r|}{ ALTERAÇÕES ALIMENTARES } \\
\hline \multicolumn{5}{|r|}{ Revisões sistemáticas } \\
\hline $\begin{array}{l}\text { Autor/ } \\
\text { Ano }\end{array}$ & $\begin{array}{l}\text { Estudos } \\
\text { incluídos }\end{array}$ & Amostra & $\begin{array}{l}\text { Critérios } \\
\text { Diagnóstico }\end{array}$ & Intervenção \\
\hline $\begin{array}{l}\text { Hall, et al } \\
(2012)^{15}\end{array}$ & $4 \mathrm{ECA}$ & $\begin{array}{l}n=22 \text { a } 267 \\
\text { Idade: inferior } \\
\text { a } 6 \text { meses }\end{array}$ & $\begin{array}{l}\text { Wessel, Wessel } \\
\text { modificados ou } \\
\text { Wessel-like }\end{array}$ & $\begin{array}{l}\text { - Fórmula enriquecida com fibra vs. placebo; } \\
\text { - Fórmula extensamente hidrolisada vs. fórmula convencional; } \\
\text { - Fórmula extensamente hidrolisada } 1 \text { vs. fórmula extensamente } \\
\text { hidrolisada } 2 \text { vs. fórmula convencional; } \\
\text { - Fórmula hidrolisada vs. fórmula convencional. } \\
\text { - Dieta materna/fórmula hipoalergénica vs. dieta ou fórmula habitual; } \\
\text { - Dieta materna hipoalergénica vs. dieta habitual. }\end{array}$ \\
\hline $\begin{array}{l}\text { lacovou, et } \\
\text { al }(2012)^{16}\end{array}$ & $13 \mathrm{ECA}$ & $\begin{array}{l}n=666 \\
\text { Idade: inferior } \\
\text { a } 6 \text { meses }\end{array}$ & $\begin{array}{l}\text { Wessel ( } 10 \\
\text { ECA) ou outros } \\
\text { (3 ECA) }\end{array}$ & $\begin{array}{l}\text { - Fórmula parcialmente hidrolisada com prébióticos ( } 14 \text { dias); } \\
\text { - Fórmula extensamente hidrolisada vs. fórmula convencional } \\
\text { (7-9 dias); } \\
\text { - Lactentes amamentados a quem foi trocada a alimentação para } \\
\text { fórmula completamente hidrolisada (4 dias); } \\
\text { - Fórmula de soja vs. fórmula convencional (2 ECA cruzados, durante } \\
\text { uma semana e } 1 \text { ECA durante } 48 \text { horas). } \\
\text { - Dieta materna hipoalergénica (eliminação leite ou derivados do leite, } \\
\text { ovos, amendoins, nozes, soja e peixe) (7-9 dias); } \\
\text { - Fórmula hipoalergénica (não sob aleitamento materno) vs. ausência } \\
\text { de intervenção ou aconselhamento parental. }\end{array}$ \\
\hline $\begin{array}{l}\text { Perry, et al } \\
(2011)^{17}\end{array}$ & $1 \mathrm{ECA}$ & $\begin{array}{l}n=29 \\
\text { Idade: } 10-54 \\
\text { dias }\end{array}$ & $\begin{array}{l}\text { Registo do } \\
\text { tempo de } \\
\text { choro }\end{array}$ & $\begin{array}{l}\text { Fórmula com polissacárido de soja ( } 14,1 \text { gramas de fibra por litro) } \\
\text { vs. fórmula convencional }\end{array}$ \\
\hline \multicolumn{5}{|r|}{ Ensaios clínicos aleatorizados e controlados } \\
\hline $\begin{array}{l}\text { Autor/ } \\
\text { IAno }\end{array}$ & Tipo & Amostra & $\begin{array}{l}\text { Critérios } \\
\text { Diagnóstico }\end{array}$ & Intervenção \\
\hline $\begin{array}{l}\text { Moravej, et } \\
\text { al (2010) }\end{array}$ & ECAC & $\begin{array}{l}n=114 \\
\text { Idade: } 3 \\
\text { semanas a } 3 \\
\text { meses (sob } \\
\text { aleitamento } \\
\text { materno } \\
\text { exclusivo) }\end{array}$ & $\begin{array}{l}\text { Critérios de } \\
\text { Wessel }\end{array}$ & $\begin{array}{l}2 \text { ramos: } \\
\text { Teste Prick cutâneo: positivo } \rightarrow \text { Eliminação do leite de vaca e } \\
\text { derivados na dieta materna; } \\
\text { Teste Prick cutâneo: negativo } \rightarrow \text { Evicção de leite e derivados vs. } \\
\text { placebo }\end{array}$ \\
\hline
\end{tabular}




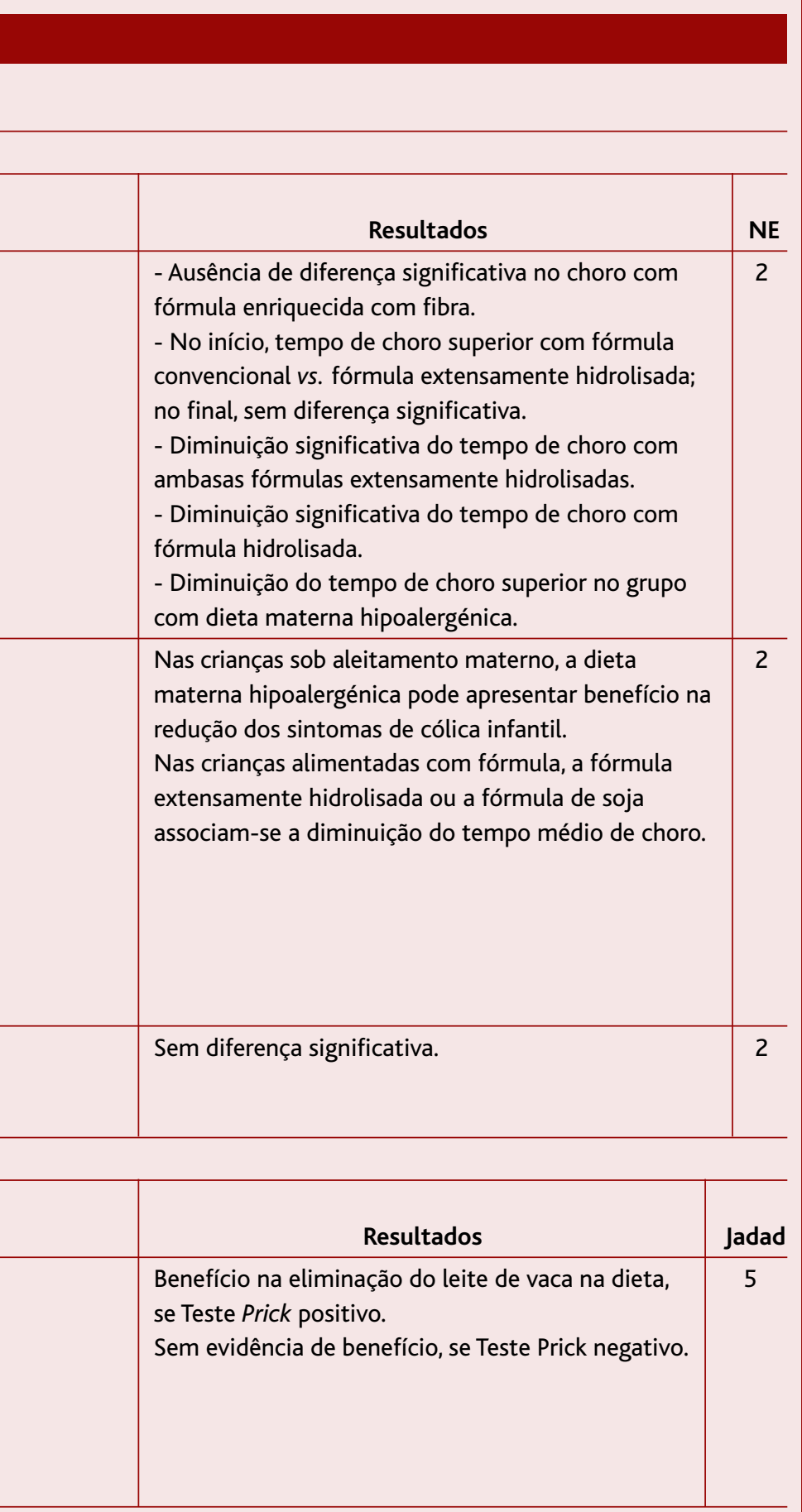

Em 2016 foram publicados os critérios de Roma IV para os distúrbios funcionais gastrointestinais, onde se incluiu também a cólica infantil. Segundo os mesmos, devem estar presentes os seguintes critérios para a definição da cólica infantil: aparecimento e desaparecimento dos sintomas antes dos cinco meses de idade; períodos de choro recorrentes e prolongados, agitação ou irritabilidade, reportados pelos cuidadores, que ocorrem sem uma causa aparente e não conseguem ser previstos ou resolvidos; e inexistência de febre, doença ou má evolução ponderal da criança. ${ }^{7}$

A etiologia permanece desconhecida, apontando-se algumas hipóteses como prováveis, nomeadamente a imaturidade intestinal, dismotilidade ou mesmo composição da flora intestinal (alguns lactobacilos podem afetar o perfil dos ácidos gordos intestinais). Alguns estudos apontam para uma possível hipersensibilidade, associada a alergia alimentar. Existe também a hipótese de associação com o tabagismo materno, devido ao aumento dos níveis plasmáticos e intestinais de motilina. Causas psicossociais, como tensão familiar, ansiedade parental ou interação inadequada também são sinalizados por alguns autores..$^{8-12} \mathrm{~A}$ maior parte dos casos provavelmente representam o pico da curva de choro do lactente saudável, não existindo evidência de que o choro se deva a dor abdominal. Porém, os pais/cuidadores frequentemente assumem que a causa do choro é a dor abdominal com origem gastrointestinal. ${ }^{7}$

As diferentes causas apontadas condicionam diferentes propostas terapêuticas, incluindo as categorias farmacológicas, alimentares ou mesmo comportamentais. Na diversidade assenta também a controvérsia, nomeadamente na tomada de decisão da melhor estratégia terapêutica a propor, tendo em conta a comprovação da sua eficácia.

Propõe-se, por isso, rever a evidência disponível sobre a eficácia das estratégias de intervenção terapêutica na cólica infantil.

\section{MÉTODOS}

A pesquisa foi referente aos artigos publicados entre 01 de janeiro de 2006 e 01 de outubro de 2016 nas seguintes bases de dados: National Guideline Clearinghouse, Canadian Medical Association Practice Guidelines Infobase, National Institute for Health and Care Excellence, The Cochrane Library, DARE, Bandolier MEDLINE. 


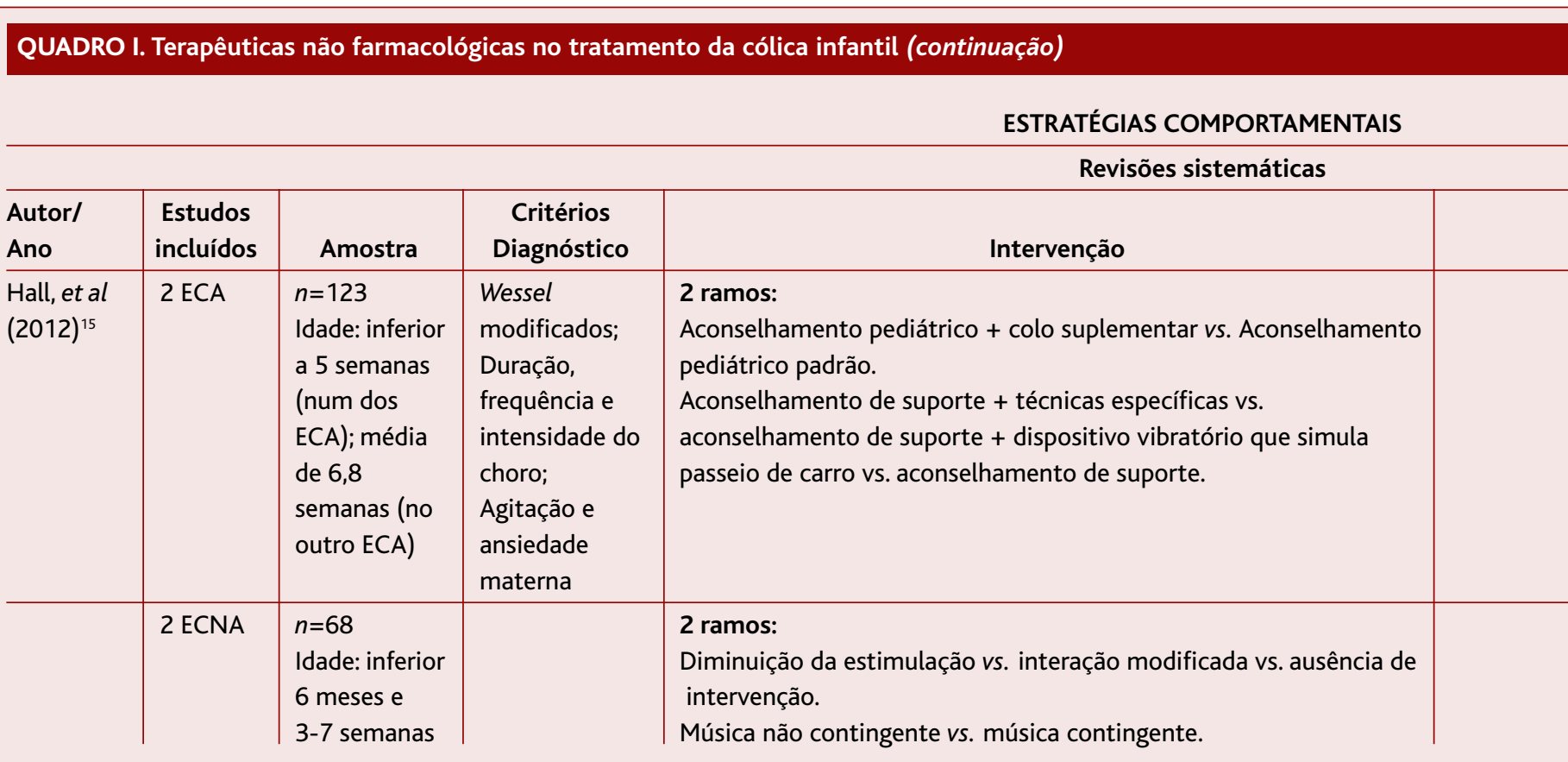






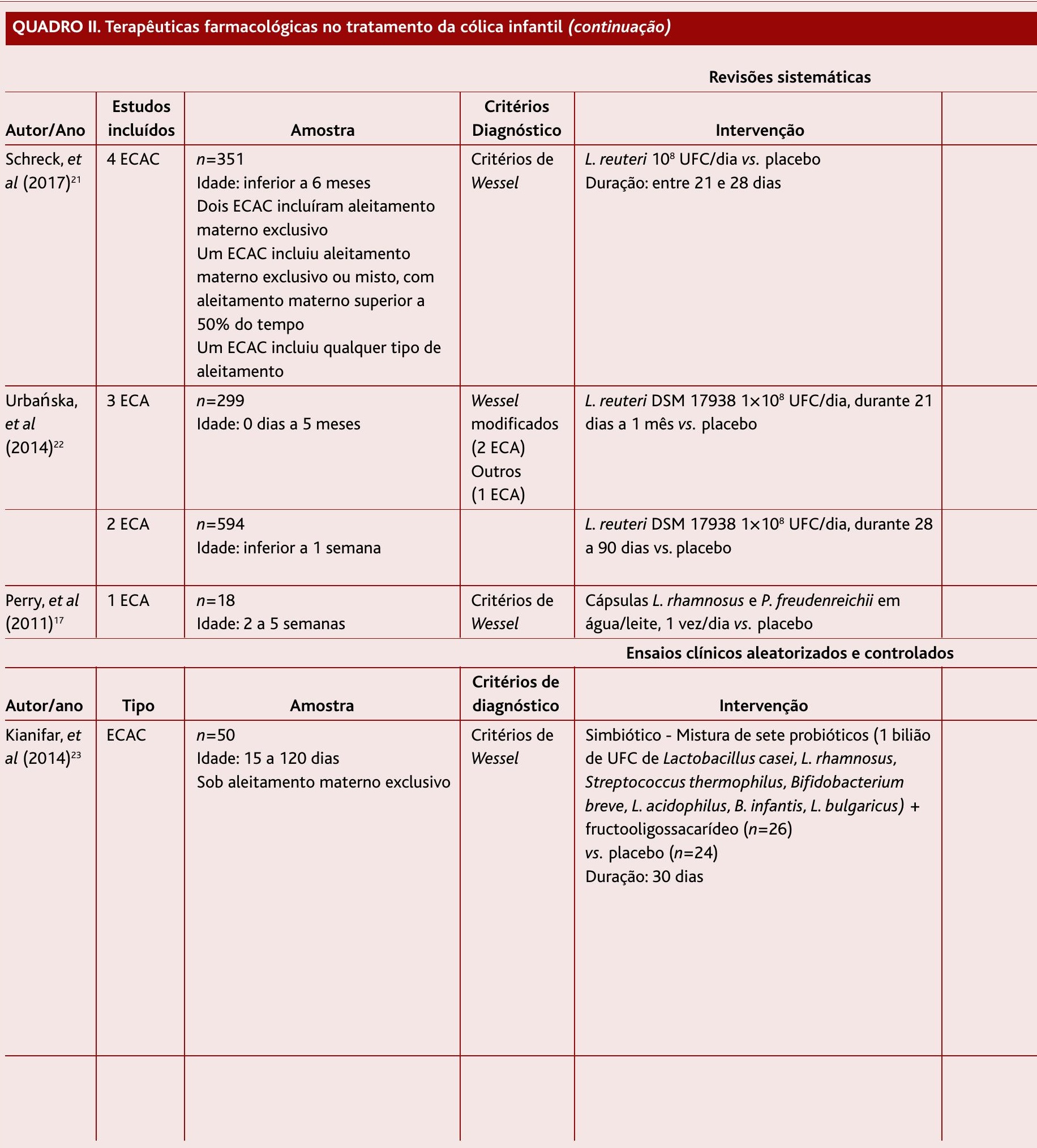




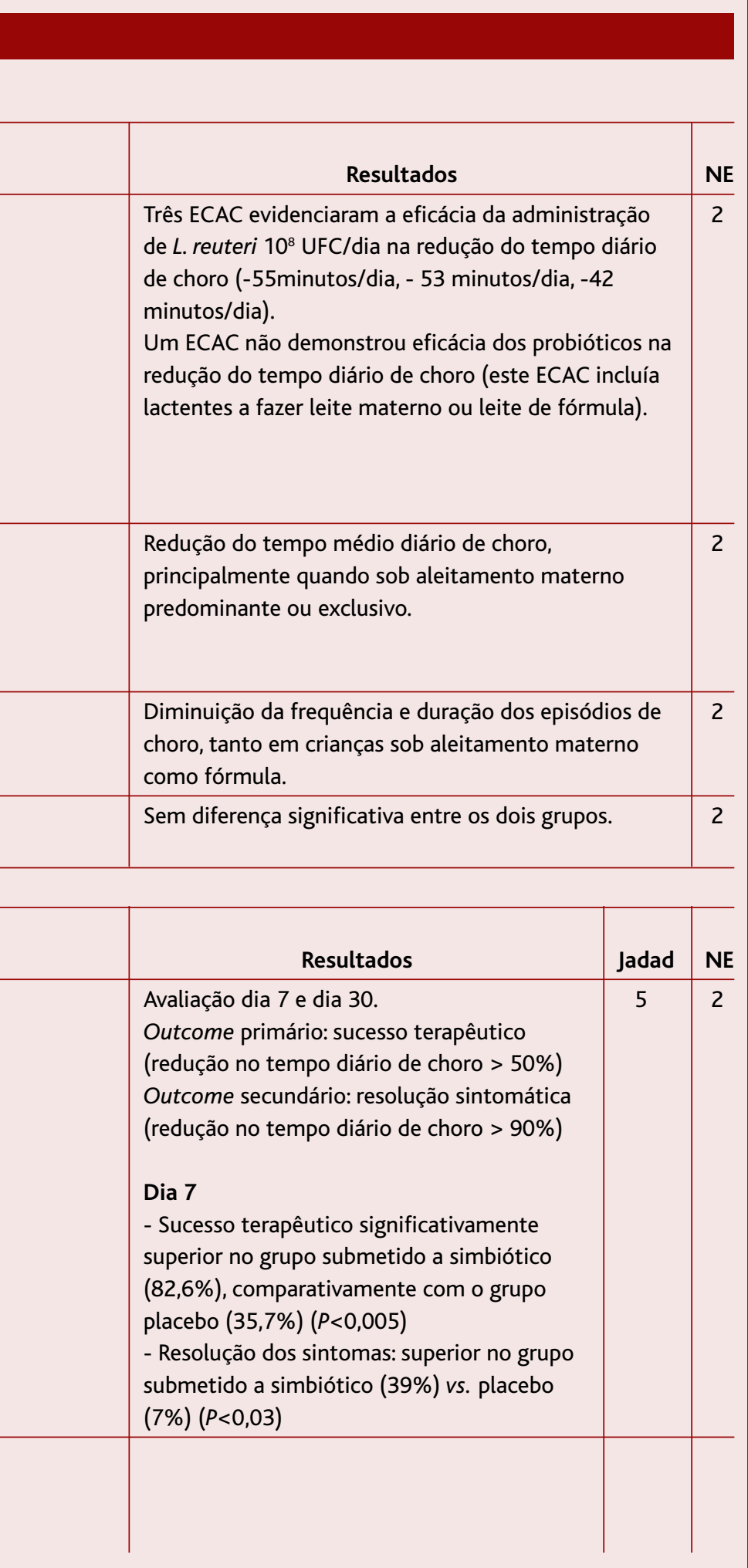

As terapias não farmacológicas são, regra geral, a primeira escolha terapêutica por parte dos pais (Quadro I).

As fórmulas láteas selecionadas, nomeadamente a hidrolisada e extensamente hidrolisada, apresentam redução significativa do tempo e número de episódios de cólica, sendo recomendadas em alternativa para casos particulares. Outras fórmulas, como fórmulas à base de soja ou enriquecidas com fibra, não demonstraram resultados significativos.

Estudos apontam que as alterações sobre a dieta materna trazem benefício nos bebés sob aleitamento materno exclusivo, adotando estratégias de uma alimentação hipoalergénica ou mesmo evicção de leite e dos seus derivados, caso a mãe apresente testes cutâneos positivos para proteína do leite de vaca.

Ao nível das estratégias comportamentais apenas a música contingente ou níveis de interação modificada, com redução de estímulos ao bebé, provaram eficácia na redução sintomática. O colo ou passeios de carro, sendo práticas comuns, não têm evidência demonstrada.

Em termos farmacológicos (Quadro II), as opções estão amplamente disseminadas entre pais, cuidadores e profissionais, sendo utilizadas de forma indiscriminada. No que aos probióticos diz respeito, são essencialmente usados os Lactobacillus (L.) reuteri ou $L$. rhammonosus, tendo apenas o primeiro demonstrado redução significativa dos episódios de cólica, merecendo recomendação na prevenção e no tratamento pelas sociedades de gastroenterologia.

Quando comparados com placebo, o simeticone, a lactase ou a sacarose/glicose não apresentam resultados na redução da cólica do lactente.

Outras terapêuticas complementares, manipulativas ou com base em fitoterápicos (Quadro III) são por vezes preteridas pelos pais em detrimento dos fármacos. Neste campo, a acupuntura apresenta resultados inconsistentes. Já as terapias manipulativas apresentam resultados consistentes na resolução da cólica infantil. Os fitoterápicos à base de funcho poderão levar à redução do tempo de choro.

\section{CONCLUSÕES}

Apesar da inclusão de 17 artigos, correspondendo a um $n$ total de grandes dimensões, alguns estudos individualmente apresentam amostras reduzidas e grande 


\begin{tabular}{|c|c|c|c|c|}
\hline & & & & Ensaios clínicos aleatorizados e controla \\
\hline Autor/ano & Tipo & Amostra & $\begin{array}{l}\text { Critérios de } \\
\text { diagnóstico }\end{array}$ & Intervenção \\
\hline $\begin{array}{l}\text { Kianifar, et } \\
\text { al }(2014)^{23}\end{array}$ & ECAC & $\begin{array}{l}n=50 \\
\text { Idade: } 15 \text { a } 120 \text { dias } \\
\text { Sob aleitamento materno exclusivo }\end{array}$ & $\begin{array}{l}\text { Critérios de } \\
\text { Wessel }\end{array}$ & $\begin{array}{l}\text { estatístico ( } 56 \% \text { vs. } 36 \%, P=0,24) \\
\text { de UFC de Lactobacillus casei, L. rhamnosus, } \\
\text { Streptococcus thermophilus, Bifidobacterium } \\
\text { breve, L. acidophilus, B. infantis, L. bulgaricus) + } \\
\text { fructooligossacarídeo }(n=26) \\
\text { vs. placebo ( } n=24) \\
\text { Duração: } 30 \text { dias }\end{array}$ \\
\hline
\end{tabular}

\section{Normas de Orientação Clínica}

\section{Nome/autor/ano}

The use of probiotics in pediatric gastroenterology: a review of the literature and recommendations by Latin-American experts Latin-American (LATAM) expert consensus group $(2015)^{24}$

Using probiotics in the paediatric population

Nutrition and Gastroenterology Committee, Canadian Paediatric Society (2012)25

Probiotics and prebiotics

World Gastroenterology Organization Global Guidelines $(2011)^{26}$

\begin{tabular}{|c|c|c|c|c|}
\hline \multicolumn{5}{|r|}{ SIMETICONE } \\
\hline \multicolumn{5}{|r|}{ Meta-análises } \\
\hline Autor/Ano & $\begin{array}{l}\text { Estudos } \\
\text { incluídos }\end{array}$ & Amostra & Critérios Diagnóstico & Intervenção \\
\hline $\begin{array}{l}\text { Biagioli, et } \\
\text { al }(2016)^{27}\end{array}$ & $2 \mathrm{ECA}$ & $\begin{array}{l}n=220 \\
\text { Idade: } 1 \text { a } 12 \text { semanas }\end{array}$ & Critérios de Wessel e outros & $\begin{array}{l}\text { - 0,3mL ( } 10 \text { gotas) antes das refeições, durante } \\
\text { uma semana, depois três dias sem tratamento } \\
\text { e novamente uma semana de tratamento } \\
\text { - 0,3mL antes das refeições, 3-10 dias } \\
\text { vs. placebo }\end{array}$ \\
\hline
\end{tabular}

\section{LACTASE}

\section{Revisões sistemáticas}

\begin{tabular}{|l|l|l|l|l|}
\hline Autor/Ano & $\begin{array}{c}\text { Estudos } \\
\text { incluídos }\end{array}$ & \multicolumn{1}{|c|}{ Amostra } & \multicolumn{1}{c|}{ Critérios Diagnóstico } & \multicolumn{1}{c}{ Intervenção } \\
\hline $\begin{array}{l}\text { Hall, et al } \\
(2012)^{15}\end{array}$ & 2 ECAC & $\begin{array}{l}n=68 \\
\text { Idade: } 3 \text { a } 13 \text { semanas } \\
\text { (média 6,4 semanas) }\end{array}$ & $\begin{array}{l}\text { Wessel-like (1 ECAC) } \\
\text { Outros (1 ECAC) }\end{array}$ & Lactase vs. placebo \\
\end{tabular}




\section{QUADRO II. Terapêuticas farmacológicas no tratamento da cólica infantil (continuação)}

SACAROSE E GLICOSE

\begin{tabular}{|c|c|c|c|c|}
\hline & & & & Meta-análises \\
\hline Autor/Ano & $\begin{array}{l}\text { Estudos } \\
\text { incluídos }\end{array}$ & Amostra & Critérios Diagnóstico & Intervenção \\
\hline $\begin{array}{l}\text { Biagioli, et } \\
\text { al }(2016)^{27}\end{array}$ & 3 ECAC & $\begin{array}{l}n=70 \\
\text { Idade média: } 2,5 \text { a } 9 \\
\text { semanas }\end{array}$ & Critérios de Wessel & $\begin{array}{l}\text { Solução Glicose } 30 \% \text { / } 1 \text { semana } \\
\text { Solução Glicose } 12 \% \text { / } 1 \text { semana ( } 2 \text { ECAC } \\
\text { vs. placebo }\end{array}$ \\
\hline
\end{tabular}

\section{QUADRO III. Terapêuticas complementares no tratamento da cólica infantil}

\section{ACUPUNTURA}

\section{Revisões sistemáticas}

\begin{tabular}{l|l|l|l|l}
\hline Autor/Ano & $\begin{array}{c}\text { Estudos } \\
\text { incluídos }\end{array}$ & \multicolumn{1}{|c|}{ Amostra } & $\begin{array}{c}\text { Critérios } \\
\text { Diagnóstico }\end{array}$ & \multicolumn{1}{c}{ Intervenção } \\
\hline $\begin{array}{l}\text { Raith, et al } \\
(2013)^{28}\end{array}$ & $3 \mathrm{ECA}$ & $\begin{array}{l}n=121 \\
\text { Idade: inferior } \\
\text { a um ano }\end{array}$ & Outros & $\begin{array}{l}\text { Acupuntura - ponto LI4 (Hegu) } \\
\text { (Quatro sessões de 20 segundos ou SEIS sessões de 2 segundos / 3 } \\
\text { semanas) vs. placebo/ausência de intervenção }\end{array}$ \\
\hline
\end{tabular}

Ensaios clínicos aleatorizados e controlados

\begin{tabular}{l|c|l|l|l|}
\hline Autor/Ano & Tipo & \multicolumn{1}{|c|}{ Amostra } & $\begin{array}{l}\text { Critérios } \\
\text { Diagnóstico }\end{array}$ & \multicolumn{1}{|c|}{ Intervenção } \\
\hline $\begin{array}{l}\text { Skjeie, et al } \\
(2013)^{29}\end{array}$ & ECAC & $\begin{array}{l}n=90 \\
\text { Idade: } 3 \\
\text { semanas a 3 } \\
\text { meses (de } \\
\text { termo) }\end{array}$ & $\begin{array}{l}\text { Critérios de } \\
\text { Wessel }\end{array}$ & $\begin{array}{l}\text { Acupuntura - ponto ST36 (quatro sessões de 30 segundos/1 semana) } \\
\text { vs. placebo/ausência de intervenção }\end{array}$ \\
\hline
\end{tabular}

\section{QUIROPRAXIA E OSTEOPATIA}

Meta-análises

\begin{tabular}{|l|l|l|l|l}
\hline Autor/Ano & $\begin{array}{l}\text { Estudos } \\
\text { incluídos }\end{array}$ & \multicolumn{1}{|c|}{ Amostra } & $\begin{array}{l}\text { Critérios } \\
\text { Diagnóstico }\end{array}$ & \multicolumn{1}{c}{ Intervenção } \\
\hline $\begin{array}{l}\text { Dobson, et } \\
\text { al (2012) }\end{array}$ & 6 ECAC & $\begin{array}{l}n=325 \\
\text { Idade: inferior } \\
\text { a } 6 \text { meses }\end{array}$ & $\begin{array}{l}\text { Critérios de } \\
\text { Wessel } \\
\text { Diários de } \\
\text { choro }\end{array}$ & $\begin{array}{l}\text { Terapias manipulativas (quiropraxia, osteopatia, osteopatia } \\
\text { craniossacral, manipulação craniana), 3 a 5 vezes por semana ou } \\
\text { on-demand, entre 10 dias a 4 semanas } \\
\text { vs. placebo/ausência de intervenção }\end{array}$ \\
\hline
\end{tabular}

\section{Revisões sistemáticas}

\begin{tabular}{|c|c|c|c|c|}
\hline Autor/Ano & $\begin{array}{l}\text { Estudos } \\
\text { incluídos }\end{array}$ & Amostra & $\begin{array}{c}\text { Critérios } \\
\text { Diagnóstico }\end{array}$ & Intervenção \\
\hline $\begin{array}{l}\text { Perry, et al } \\
(2011)^{17}\end{array}$ & $3 \mathrm{ECA}$ & $\begin{array}{l}n=149 \\
\text { Idade: } 0 \text { a } 9 \\
\text { semanas } \\
\text { (média } 45 \\
\text { dias) }\end{array}$ & $\begin{array}{l}\text { Wessel } \\
(1 \mathrm{ECA}) \\
\text { Outros } \\
(2 \mathrm{ECA})\end{array}$ & $\begin{array}{l}\text { Osteopatia craniana, } 1 x / \text { semana, durante } 4 \text { semanas ( } 1^{\text {a }} \text { sessão: } \\
1 \text { hora e subsequentes } 30 \text { minutos) } \\
\text { Manipulação espinhal quiropráxica (sessões } 10 \text { minutos, máximo } 6 \\
\text { tratamentos } / 2 \text { semanas. OU } 3 x / \text { semana, durante } 8 \text { dias) vs. } \\
\text { placebo/ausência de intervenção }\end{array}$ \\
\hline
\end{tabular}




\section{Resultados}

Apenas um estudo de baixa qualidade demonstrou redução tempo de choro diário (diferença média de 1,72 horas; IC95\% 1,38-2,06).

\begin{tabular}{l|l|c}
\hline \multicolumn{1}{|c|}{ Resultados } & NE \\
\hline & $\begin{array}{l}\text { Diminuição da frequência e intensidade do choro. } \\
\text { Ausência de diferença significativa nos padrões de } \\
\text { alimentação, dejeções ou sono. }\end{array}$ & 2 \\
\hline
\end{tabular}

\begin{tabular}{l|l|c|c}
\hline \multicolumn{1}{|c|}{ Resultados } & Jadad & NE \\
\hline $\begin{array}{l}\text { Sem diferença estatisticamente significativa, } \\
\text { apesar de diminuição do choro diário em 13 } \\
\text { minutos. }\end{array}$ & 5 & 2 \\
\hline
\end{tabular}

\begin{tabular}{|l|l|c} 
& \multicolumn{1}{|c}{ Resultados } & NE \\
\hline & $\begin{array}{l}\text { Diminuição do tempo de choro (diferença média de 1 } \\
\text { hora e 12 minutos; IC95\% -1,89; a -0,51, com } \\
\text { significado estatístico). }\end{array}$ & 2 \\
\hline & \multicolumn{1}{|c}{ Resultados } & NE \\
\hline & $\begin{array}{l}\text { Diminuição significativa do tempo de choro diário } \\
\text { [diferença média de 1 hora/dia (IC95\% 0,14-2,19)]. } \\
\text { Aumento significativo do tempo de sono [1 hora e } \\
10 \text { minutos /dia (IC95\% 0,29-2,27)]. } \\
\text { Resolução completa dos sintomas (93\% das crianças), } \\
\text { sem recorrência da cólica ao fim de um mês. }\end{array}$ & 2 \\
\hline
\end{tabular}

das dependerem de observações individuais e subjetivas, de não existirem critérios objetivos e de se tratar de uma população de recém-nascidos e lactentes, dificulta a realização de ensaios clínicos.

Os pais, que regra geral aplicam as intervenções e repostam os resultados, deveriam ser sempre ocultados do efeito terapêutico vs. placebo. Tendo em conta que é um processo fisiológico e autolimitado, na ausência de evidência robusta sobre a eficácia dos tratamentos, a atitude expectante pode ser uma estratégia a considerar.

\section{REFERÊNCIAS BIBLIOGRÁFICAS}

1. Lucassen PL, Assendelft WJ, van Eijk JT, Gubbels JW, Douwes AC, van Geldrop WJ. Systematic review of the occurrence of infantile colic in the community. Arch Dis Child. 2001;84(5):398-403.

2. Akhnikh S, Engelberts AC, van Sleuwen BE, L'Hoir MP, Benninga MA. The excessively crying infant: etiology and treatment. Pediatr Ann. 2014;43(4):e69-75.

3. Kurth E, Kennedy HP, Spichiger E, Hösli I, Stutz EZ. 'Crying babies, tired mothers: what do we know? A systematic review'. Midwifery. 2011;27 (2):187-94.

4. Radesky JS, Zuckerman B, Silverstein M, Rivara FP, Barr M, Taylor JA, et al. Inconsolable infant crying and maternal postpartum depressive symptoms. Pediatrics. 2013;131(6):e1857-64.

5. Barr RG, Trent RB, Cross J. Age-related incidence curve of hospitalized Shaken Baby Syndrome cases: convergent evidence for crying as a trigger to shaking. Child Abuse Negl. 2006;30(1):7-16.

6. Wessel MA, Cobb JC, Jackson EB, Harris GS Jr, Detwiler AC. Paroxysmal fussing in infancy, sometimes called colic. Pediatrics. 1954;14(5):42135.

7. Benninga MA, Nurko S, Faure C, Hyman PE, Roberts IS, Schechter NL. Childhood functional gastrointestinal disorders: neonate/toddler. Gastroenterology. 2016;150(6):1443-55.e2

8. Ali AM. Helicobacter pylori and infantile colic. Arch Pediatr Adolesc Med. 2012;166(7):648-50.

9. SungV, Hiscock H, Tang ML, Mensah FK, Nation ML, Satzke C, et al. Treating infant colic with the probiotic Lactobacillus reuteri: double blind, placebo controlled randomised trial. BMJ. 2014;348:g2107.

10. Kurtoglu S, Uzüm K, Hallac IK, Coskum A. 5-Hydroxy-3-indole acetic acid levels in infantile colic: is serotoninergic tonus responsible for this problem? Acta Paediatr. 1997;86(7):764-5.

11. Søndergaard C, Henriksen TB, Obel C, Wisborg K. Smoking during pregnancy and infantile colic. Pediatrics. 2001;108(2):342-6.

12. Rhoads JM, Fatheree NY, Norori J, Liu Y, Lucke JF, Tyson JE, et al. Altered fecal microflora and increased fecal calprotectin in infants with colic. J Pediatr. 2009;155(6):823-8.e1.

13. Jadad AR, Moore RA, Carroll D, Jenkinson C, Reynolds DJ, Gavaghan DJ, et al. Assessing the quality of reports of randomized clinical trials: Is blinding necessary? Control Clin Trials. 1996;17(1):1-12.

14. Ebell MH, Siwek J, Weiss BD, Woolf SH, Susman J, Ewigman B, et al. Strength of recommendation taxonomy (SORT): a patient-centered ap- 


\begin{tabular}{|c|c|c|c|c|}
\hline \multirow[b]{2}{*}{ Autor/Ano } & \multirow[b]{2}{*}{$\begin{array}{l}\text { Estudos } \\
\text { incluídos }\end{array}$} & \multirow[b]{2}{*}{ Amostra } & \multicolumn{2}{|r|}{ Revisões sistemáticas } \\
\hline & & & \multicolumn{2}{|l|}{$\begin{array}{l}\text { Critérios } \\
\text { Diagnóstico }\end{array}$} \\
\hline $\begin{array}{l}\text { Bronfort, et } \\
\text { al }(2010)^{31}\end{array}$ & $4 \mathrm{ECA}$ & & Wessel & $\begin{array}{l}\text { Efeito de diversas terapias manuais (entre as quais a quiropraxia) } \\
\text { vs. placebo }\end{array}$ \\
\hline \multicolumn{5}{|r|}{ FITOTERAPIA } \\
\hline \multicolumn{5}{|r|}{ Meta-análises } \\
\hline Autor/Ano & $\begin{array}{l}\text { Estudos } \\
\text { incluídos }\end{array}$ & Amostra & $\begin{array}{l}\text { Critérios } \\
\text { Diagnóstico }\end{array}$ & Intervenção \\
\hline $\begin{array}{l}\text { Biagioli, et } \\
\text { al }(2016)^{27}\end{array}$ & $4 \mathrm{ECA}$ & $\begin{array}{l}n=344 \\
\text { Idade: } 2 \text { a } 25 \\
\text { semanas }\end{array}$ & $\begin{array}{l}\text { Critérios de } \\
\text { Wessel ( } 3 \mathrm{ECA}) \\
\text { e outros } \\
(1 \mathrm{ECA})\end{array}$ & $\begin{array}{l}\text { 150mL de chá de ervas (camomila, funcho, verbena, alcaçuz, menta) } \\
\text { em cada episódio de cólica / uma semana } \\
\text { ColiMil }{ }^{\circledR} \text { (funcho, camomila, erva cidreira), } 2 \mathrm{~mL} / \mathrm{kg} / \mathrm{dia}, 2 \text { x/dia } \\
\text { (entre as } 17 \text { horas e as } 20 \text { horas, pré-mamada) / uma semana } \\
\text { Emulsão de óleo de funcho a 0,1\% ( } 5 \mathrm{~mL}-20 \mathrm{~mL} \text { ) } 4 \text { vezes/dia, antes } \\
\text { das refeições (máximo } 12 \mathrm{~mL} / \mathrm{Kg} / \mathrm{dia} \text { ) / uma semana }\end{array}$ \\
\hline
\end{tabular}

\begin{tabular}{|c|c|c|c|c|}
\hline \multicolumn{2}{|c|}{ Terapêutica } & Fundamento & NE & FR \\
\hline \multirow[t]{4}{*}{ Alterações alimentares } & Fórmula extensamente hidrolisada & $\begin{array}{l}\text { Hall, et al }(2012)^{15} \\
\text { lacovou, et al }(2012)^{16}\end{array}$ & $\begin{array}{l}2 \\
2\end{array}$ & B \\
\hline & Fórmula hidrolisada & Hall, et al (2012) ${ }^{15}$ & 2 & B \\
\hline & Dieta materna hipoalergénica & Hall, et al (2012) ${ }^{15}$ & 2 & B \\
\hline & $\begin{array}{l}\text { Eliminação de leite de vaca e derivados da } \\
\text { dieta materna (teste de Prick positivo) }\end{array}$ & Moravej (2010)18 & 2 & B \\
\hline \multirow[t]{2}{*}{ Estratégias comportamentais } & Interação modificada & Hall, et al (2012) ${ }^{15}$ & 2 & B \\
\hline & Música contingente & Hall, et al (2012) ${ }^{15}$ & 2 & B \\
\hline Probióticos & L. reuteri & $\begin{array}{l}\text { Xu, et al }(2015)^{19} \\
\text { Schreck, et al }(2017)^{21} \\
\text { Urbańska, et al }(2014)^{22} \\
\text { Anabrees, et al }(2013)^{20} \\
\text { LATAM }(2015)^{24} \\
\text { Canadian Paediatric Society }(2012)^{25} \\
\text { World Gastroenterology Org. }(2012)^{26}\end{array}$ & $\begin{array}{l}1 \\
2 \\
2 \\
2 \\
1 \\
3 \\
1\end{array}$ & A \\
\hline Acupuntura & Ponto LI4 (Hegu) & Raith, et al (2012) $)^{28}$ & 2 & C \\
\hline Quiropraxia/Osteopatia & & $\begin{array}{l}\text { Dobson, et al }(2012)^{30} \\
\text { Perry, et al }(2011)^{17}\end{array}$ & $\begin{array}{l}2 \\
2\end{array}$ & B \\
\hline Fitoterapia & $\begin{array}{l}\text { Chá de ervas } \\
\text { Extrato de plantas } \\
\text { Óleo de funcho }\end{array}$ & Biagioli, et al $(2016)^{27}$ & 2 & B \\
\hline
\end{tabular}




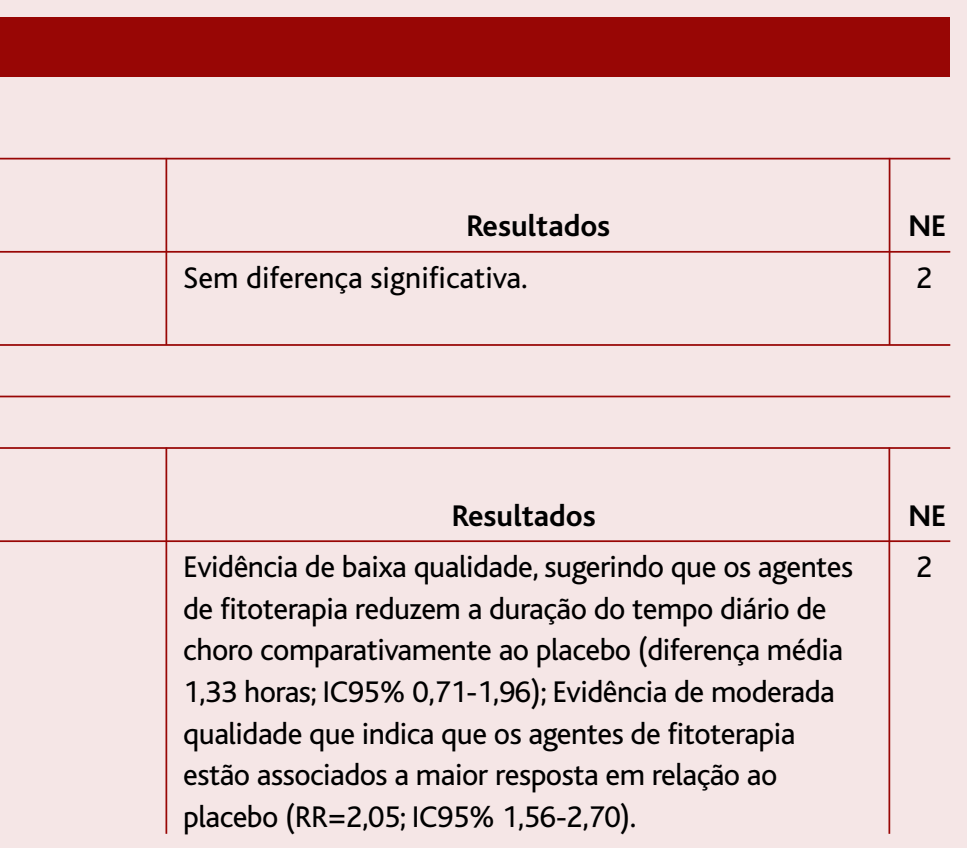

proach to grading evidence in the medical literature. Am Fam Physician. 2004;69(3):548-56.

15. Hall B, Chesters J, Robinson A. Infantile colic: a systematic review of medical and conventional therapies. J Paediatr Child Health. 2012;48(2):128-37.

16. Iacovou M, Ralston RA, Muir J, Walker KZ, Truby H. Dietary management of infantile colic: a systematic review. Matern Child Health J. 2012;16(6):1319-31.

17. Perry R, Hunt K, Ernst E. Nutritional supplements and other complementary medicines for infantile colic: a systematic review. Pediatrics. 2011;127(4):720-33.

18. Moravej $\mathrm{H}$, Imanieh MH, Kashef S, Handjani F, Eghterdari F. Predictive value of the cow's milk skin prick test in infantile colic. Ann Saudi Med. 2010;30(6):468-70.

19. Xu M, Wang J, Wang N, Sun F, Wang L, Liu XH. The efficacy and safety of the probiotic bacterium Lactobacillus reuteri DSM 17938 for infantile colic: a meta-analysis of randomized controlled trials. PLoS One. 2015;10(10):e0141445.

20. Anabrees J, Indrio F, Paes B, AlFaleh K. Probiotics for infantile colic: a systematic review. BMC Pediatr. 2013;13:186.

21. Schreck Bird A, Gregory PJ, Jalloh MA, Risoldi Cochrane Z, Hein DJ. Probiotics for the treatment of infantile colic: a systematic review. J Pharm Pract. 2017;30(3):366-74.

22. Urba ska M, Szajewska $H$. The efficacy of Lactobacillus reuteri DSM 17938 in infants and children: a review of the current evidence. Eur J Pediatr. 2014;173(10):1327-37.

23. Kianifar $H$, Ahanchian $H$, Grover Z, Jafari S, Noorbakhsh Z, Khakshour $A$, et al. Synbiotic in the management of infantile colic: a randomised controlled trial. J Paediatr Child Health. 2014;50(10):801-5.

24. Cruchet S, Furnes R, Maruy A, Hebel E, Palacios J, Medina F, et al. The use of probiotics in pediatric gastroenterology: a review of the literature and recommendations by Latin-American experts. Paediatr Drugs. 2015;17(3):199-216.

25. Marchand V. Using probiotics in the paediatric population. Paediatr Child Health. 2012;17(10):575-6.

QUADRO V. Estratégias que não parecem ter evidência no tratamento da cólica infantil

\begin{tabular}{|c|c|c|c|c|}
\hline \multicolumn{2}{|c|}{ Terapêutica } & \multirow{2}{*}{$\begin{array}{r}\text { Fundamento } \\
\text { Hall, et al (2012) }\end{array}$} & \multirow{2}{*}{$\frac{\mathrm{NE}}{2}$} & \multirow{2}{*}{$\begin{array}{c}\mathrm{FR} \\
\mathrm{B}\end{array}$} \\
\hline Alterações alimentares & Fórmula enriquecida em fibra & & & \\
\hline & Fórmula de soja & Perry, et al (2012) ${ }^{17}$ & 2 & B \\
\hline \multirow[t]{3}{*}{ Estratégias comportamentais } & Colo suplementar & Hall, et al (2012) ${ }^{15}$ & 2 & B \\
\hline & Passeio de carro & Hall, et al (2012) ${ }^{15}$ & 2 & B \\
\hline & Menor estimulação & Hall, et al (2012) ${ }^{15}$ & 2 & B \\
\hline Probióticos & L. rhamnosus e P. freudenreichii & Perry, et al (2011) ${ }^{17}$ & 2 & B \\
\hline Simeticone & & Biagioli, et al $(2016)^{27}$ & 2 & B \\
\hline Lactase & & Hall, et al (2012) ${ }^{15}$ & 2 & B \\
\hline Sacarose/glicose & & Biagioli, et al $(2016)^{27}$ & 3 & $\mathrm{C}$ \\
\hline
\end{tabular}


26. Guarner F, Khan AG, Garisch J, Eliakim R, Gangl A, Thomson A, et al. World Gastroenterology Organisation Global Guidelines: probiotics and prebiotics October 2011. J Clin Gastroenterol. 2012;46(6):468-81.

27. Biagioli E, Tarasco V, Lingua C, Moja L, Savino F. Pain-relieving agents for infantile colic. Cochrane Database Syst Rev. 2016;9:CD009999.

28. Raith W, Urlesberger B, Schmölzer GM. Efficacy and safety of acupuncture in preterm and term infants. Evid Based Complement Alternat Med. 2013;2013:739414.

29. Skjeie H, Skonnord T, Fetveit A, Brekke M. Acupuncture for infantile colic: a blinding-validated, randomized controlled multicentre trial in general practice. Scand J Prim Health Care. 2013;31(4):190-6.

30. Dobson D, Lucassen PL, Miller JJ, Vlieger AM, Prescott P, Lewith G. Manipulative therapies for infantile colic. Cochrane Database Syst Rev. 2012;12:CD004796.

31. Bronfort G, Haas M, Evans R, Leininger B, Triano J. Effectiveness of manual therapies: the UK evidence report. Chiropr Osteopat. 2010;18:3.

\section{CONFLITO DE INTERESSES}

Os autores declaram não ter quaisquer conflitos de interesse.

\section{FONTES DE FINANCIAMENTO}

Os autores declaram não ter beneficiado de quaisquer fontes externas de financiamento para a realização deste artigo.

\section{APRESENTAÇÕES E PRÉMIOS}

Versão anterior do trabalho apresentada sob a forma de comunicação oral no XXII Encontro do Internato de Medicina Geral e Familiar da Zona Norte (Porto, 12.10.2015).

\section{ENDEREÇO PARA CORRESPONDÊNCIA}

Ana Clara Moreira

E-mail: ana.cl.moreira@gmail.com

https://orcid.org/0000-0002-7838-9971

Recebido em 17-06-2017

Aceite para publicação em 12-05-2019

\section{ABSTRACT}

\section{TREATMENT OF INFANTILE COLIC: AN EVIDENCE-BASED REVIEW}

Objectives: The aim is to review the effectiveness of therapeutic interventions in infantile colic, since it is a frequent reason for consultation, its treatment is challenging, and it is a source of frustration for parents and health care professionals.

Data sources: National Guideline Clearinghouse, Canadian Medical Association Practice Guidelines Infobase, NICE, Cochrane, DARE, Bandolier and MEDLINE.

Methods of review: We searched for meta-analysis (MA), systematic reviews (SR), randomised controlled trials (RCT), clinical guidelines, and other original studies published in Portuguese, English and Spanish between January 2006 and October 2016, using the MeSH term 'infantile colic'. The Jadad Scale was used to assess the quality of clinical trials and the Strength of Recommendation Taxonomy Scale (SORT) for assigning the level of evidence and strength of recommendation.

Results: Of the 185 articles obtained, 17 met the inclusion criteria (four MA, seven SR, three RCT and three guidelines). Almost all articles evaluated crying time as the primary outcome. Hydrolysed formulas have demonstrated efficacy, but should not be used indiscriminately. The evidence is more consistent about the use of probiotics (L. reuteri DSM 17938), which showed a reduction in the daily mean crying time, either in preventive or therapeutic regimen. Simethicone, that is frequently used, did not show benefits. In complementary therapies, there is some evidence in favor of the use of fennel-based herbal medicine.

Conclusions: There are some strategies that seem to present benefit in the treatment of infantile colic, however the evidence is poor and inconsistent. Further studies with larger samples and standardised diagnostic criteria are needed. Since it is a selflimiting process, in the absence of robust evidence on the effectiveness of treatments, expectant management may be a strategy to consider.

Keywords: Infantile colic; Newborn; Infant; Primary health care. 